\title{
A Proposed Method to Reduce the BER in Wavelet based OFDM
}

\author{
Noura Ali \\ High tech. institute \\ $10^{\text {th }}$ of Ramadan \\ Egypt
}

\author{
M. Ibraheem \\ Al-Azhar University \\ Cairo \\ Egypt
}

\author{
I. Fathi \\ Al-Azhar \\ Cairo \\ Egypt
}

\begin{abstract}
Long term evolution (LTE) selects orthogonal frequency division multiplexing (OFDM) and its single-carrier counterpart SC-FDM as the basic transmission schemes. OFDM is a very interesting approach for high data rate transmission in a Gaussian channel and multipath fading environment that leads to inter symbol interference (ISI). In this paper, a proposed convolutional encoder model is used to improve the error rate performance of OFDM system, by using discrete wavelet transform (DWT) instead of fast Fourier transform (FFT) to reduce ISI. The performance of OFDM based on DWT using M-ary quadrature amplitude modulation (M-QAM) as a modulation scheme to achieve high data rate. The simulation results demonstrate that, the bit error rate (BER) is improved for a proposed convolutional encoder model as compared with the previous work.
\end{abstract}

\section{General Terms}

LTE, MATLAB simulator

\section{Keywords}

LTE, OFDM, SC-FDMA, ISI, DWT, FFT, M-QAM, BER

\section{INTRODUCTION}

LTE is a technology that supports high data rates in mobile communication, which uses the OFDM technology in download as a modulation technique which allocates orthogonal subcarriers and consider as a kind of multi-carrier modulation. OFDM divides the available spectrum into a number of parallel subcarriers and each subcarrier is then modulated by a low rate data stream at different carrier frequency. FFT based OFDM uses the cyclic prefix (CP) to reduce the problems of inter carrier interference (ICI) and ISI that consume $25 \%$ from the channel bandwidth. The use of wavelet allows reducing the ISI and ICI without the usage of cyclic prefix and also save $25 \%$ of bandwidth. Wavelet transform analyzes the signal in both time and frequency domain. The properties of wavelets make it as a better choice for different applications like biomedical engineering, nuclear engineering, pure mathematics, computer graphics and animation, earthquakes prediction, image processing, data compression, music, optics, human vision, radar etc, the wavelet has many functions such Haar, daubechies, coiflet, biorthogonal, reverse biorthogonal and symlet etc. Haar wavelet transform has some advantages over the other wavelet functions such as simplicity, fast, easy and memory efficient. This paper review the FFT based OFDM, wavelet based OFDM and channel coding, after that the proposed convolutional encoder are studied and last the simulation results. The results in this paper compare the wavelet with FFT based OFDM and the different wavelet functions. The last part compares between the system performances in case of no coding applying and applies the convolutional coding and the proposed model, lastly how to increase the data rates.

\section{FFT BASED OFDM SYSTEM}

The block diagram of FFT based OFDM system is shown in Fig.1. The input digital data is processed by M-QAM to map the data with $\mathrm{N}$ subcarriers that are implemented using the IFFT block. After symbol mapping, it is necessary to convert the data stream into parallel form, so a serial to parallel converter is used. IFFT block is then used to modulate this low data rate stream that also converts the domain of the input. The output of IFFT is the sum of the information signals in the discrete time domain as follows [1]:

$x_{k}=\frac{1}{N} \sum_{m=0}^{N-1} X_{m} e^{j 2 \pi k m / N}$

Where, $x_{k}(0<k<N-1)$ is a sequence data in a discrete time domain, $X_{m}$ is complex number in a discrete frequency domain and $\mathrm{N}$ is the number of subcarriers After applying IFFT on the symbols in all the channels, cyclic prefix is added. The addition of a cyclic prefix to each symbol solves for both the Inter Symbol Interference and Inter Carrier Interference. Digital data is converted to serial form and transmitted over the channel. At the receiver side, the process is reversed to obtain the data. The output of FFT is represented as follow [1]:

$X_{m}=\sum_{k=0}^{N-1} x_{k} e^{-j 2 \pi k m / N}$

After FFT, the signal is converted to parallel form by using serial to parallel converter and demodulated by m-ary QAM demodulator to obtain the output binary data.

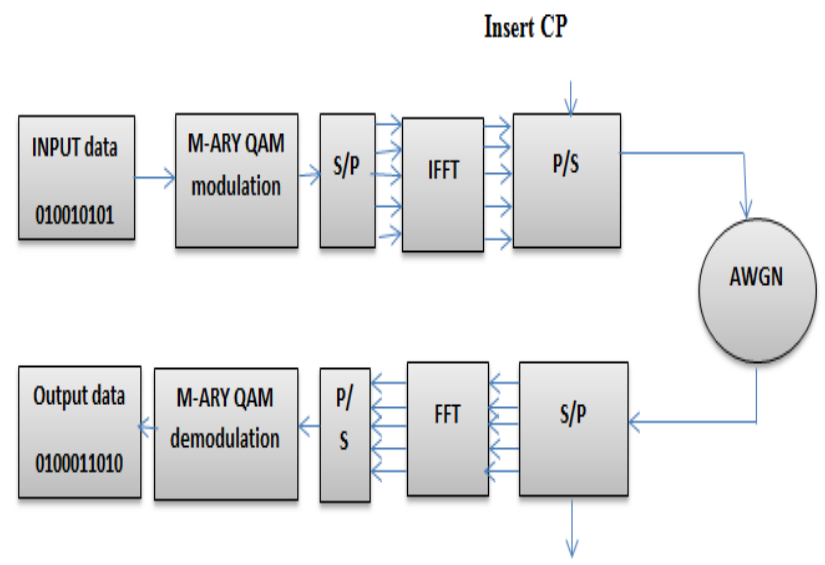

Remove CP

Fig. 1 Block diagram of FFT based OFDM transceiver 


\section{WAVELET BASED OFDM SYSTEM}

The block diagram of wavelet based OFDM is shown in Fig. 2. The inverse discrete wavelet transform (IDWT) used in the transmitter gives the following output:

$d(k)=\sum_{m=0}^{\infty} \sum_{n=0}^{\infty} D_{m}^{n} \frac{2^{m}}{2} \varphi\left(2_{k}^{m}-n\right)$

Where $\mathrm{D}_{\mathrm{m}}^{\mathrm{n}}$ are the wavelet coefficients and $\varphi(t)$ is the wavelet function with compressed factors $m$ and $n$ for each subcarriers number $\mathrm{k}(0 \leq \mathrm{k} \leq \mathrm{N}-1)$. At the receiver side, the process is inversed. The output of discrete wavelet transform (DWT) is

$D_{m}^{n}=\sum_{k=0}^{N-1} d(k) \frac{2^{m}}{2} \varphi\left(2_{k}^{m}-n\right)$

In the transmitter, data are generated in the random binary digital form. The QAM modulation converts the binary data into modulated symbols. These symbols and zeros pads are converted parallel to serial and then apply to vector transpose. The output of the vector transpose is considered as approximated coefficients and detailed coefficients, respectively. This whole part is also known as synthesis.

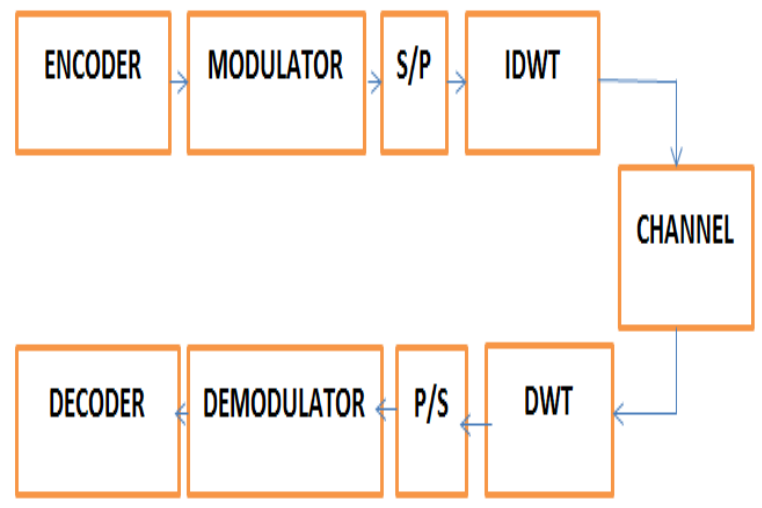

Fig. 2 The block diagram of Wavelet based OFDM transceiver

\section{CHANNEL CODING}

The main channel coding types are the block coding, convolutional coding and turbo coding [2]. Convolutional encoders are very powerful error correcting codes which finds their applications in digital video, satellite communication and mobile communication. Convolutional encoder is finite state machine which processes the information bits in a serial manner and applied in applications of good performance and low implementation cost. Convolutional encoders are denoted by $(\mathrm{n}, \mathrm{k}, \mathrm{L})$, where $\mathrm{L}$ is code (or encoder) Memory depth (number of memory register stages), $\mathrm{k}$ is the input bits and $\mathrm{n}$ is output bits as shown in Fig. 3 [3].

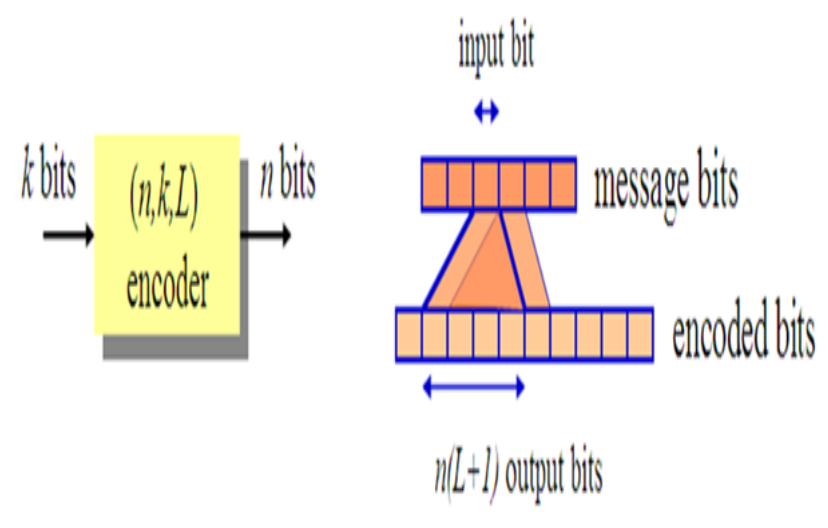

Fig. 3 The convolutional encoder

Constraint length $\mathrm{C}=\mathrm{n}(\mathrm{L}+1)$ is defined as the number of the encoded bits. $\mathrm{k} / \mathrm{n}$ is the code rate and is a measure of efficiency of the code .

\section{PROPOSED CONVOLUTIONAL ENCODER MODEL}

The convolutional encoder enhances the OFDM performance by enhancing the system performance. The proposed convolutional encoder model used in this paper generates a random binary data to pass through two convolutional encoder blocks. The encoded data interleaved by a matrix interleaver to be modulated via a QAM modulator block. The proposed model used the IDWT for the transformation in transmitter a shown in Fig.4.

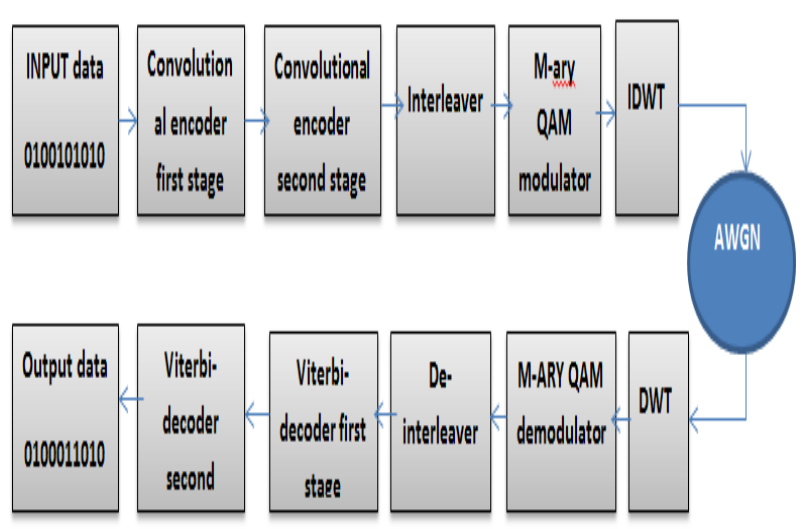

Fig, 4 Theproposed conrolutionalencodermodel transceiver

The receiver makes the reverse process after passing the transformed signal through the additive white Gaussian noise (AWGN) channel. The proposed model used a two stage of Viterbi decoder with the convolutional encoder to obtain high performance and simplicity.

\section{SIMULATION RESULTS}

The simulation of wavelet based OFDM and FFT based OFDM are compared for 16-QAM modulator. The simulation results are implemented using MATLAB 
(v8.2015a) software. The results plotted for AWGN channel because of its simplicity than the multipath and Rayleigh channel [4]. BER curves are plotted versus the signal to noise ratio $(\mathrm{S} / \mathrm{N})$ in $\mathrm{dB}$ for different cases of wavelet based OFDM. The system works with 9600 bits for each frames, $N=512$ FFT size, 16-QAM modulation and $10 \mathrm{MHz}$ bandwidth with carrier spacing of $15 \mathrm{kHz}$.

\subsection{Comparing BER in wavelet and FFT based OFDM}

Comparing the BER performance for FFT based OFDM with inserting a cyclic prefix and Wavelet based OFDM without any cyclic prefix and using a Haar wavelet function [5 - 6]. BER value equal 0.1454 in FFT based OFDM at $10 \mathrm{~dB}$ as shown in Fig. 5 whereas equal 0.002604 in wavelet based OFDM at the same signal to noise ratio $10 \mathrm{~dB}$ as shown in Fig. 6. These results gives a better performance of wavelet based OFDM than FFT based OFDM [7]. The results give an enhancement for wavelet based OFDM than FFT based OFDM by reducing the BER value at the same signal to noise ratio. The reduction in BER value gives low errors and high accuracy and performance in the system.

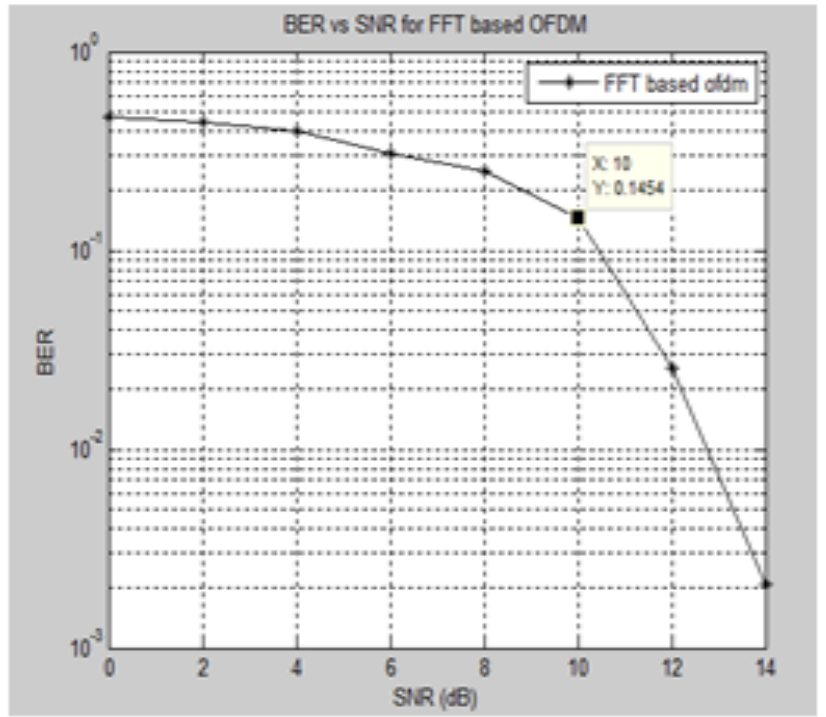

Fig 5 BER in FFT based OFDM

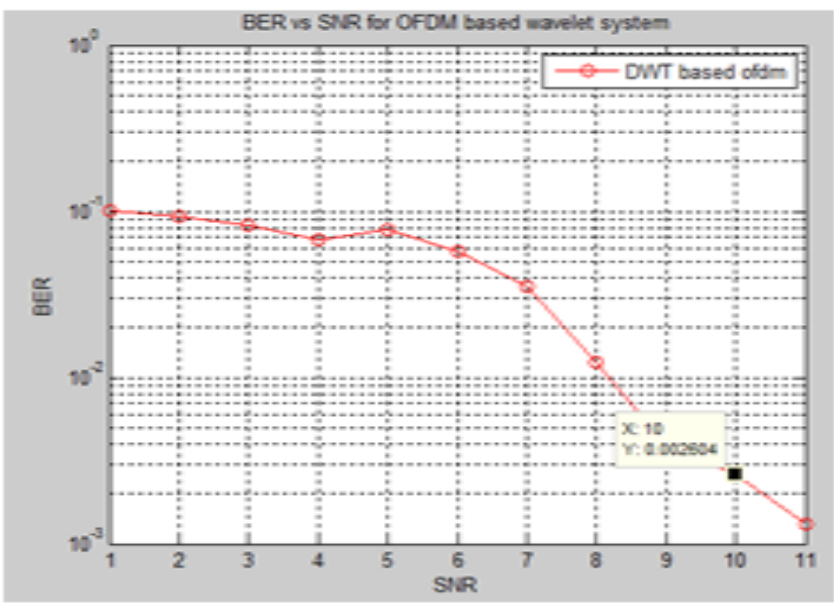

Fig 6 BER in wavelet based OFDM

\subsection{BER in wavelet based OFDM system for different wavelet functions}

The simulation of wavelet based OFDM for different wavelet functions are studied in this section. The wavelet has many functions such Haar, daubechies, coiflet, biorthogonal, reverse biorthogonal and symlet etc [8]

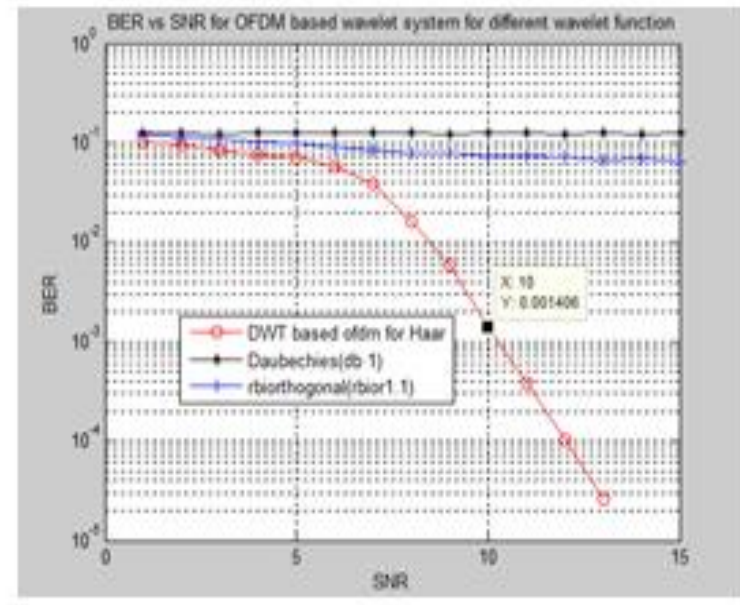

\section{Fgg 7 BER forvanous wavelet finctions}

\subsection{Effect of Convolutional coding in wavelet based OFDM}

This section shows the effect of system before applying the convolutional channel coding and after applying it. The enhancement in the system performance can be obtained by reducing the BER for the same $\mathrm{S} / \mathrm{N}$ ratio. The system without any coding gives a BER value of 0.08681 at $10 \mathrm{~dB}$ as shown in Fig. 8.

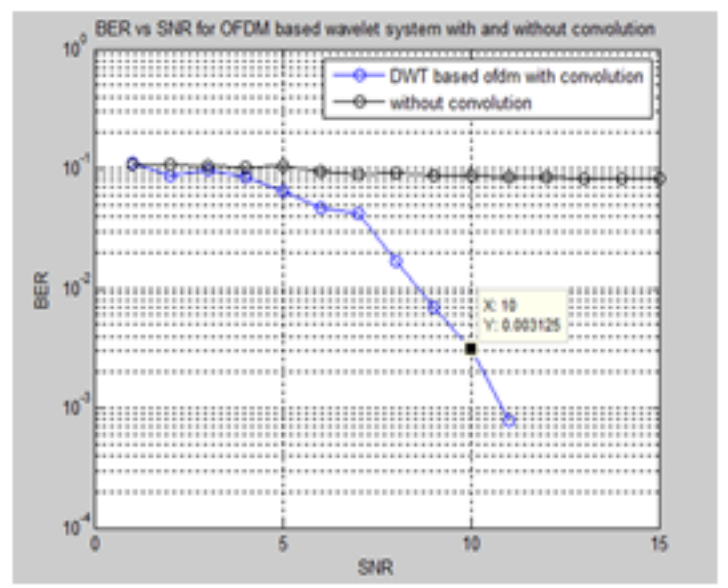

Fig 8Wavelet basedOFDM with and without coding

When applying a convolutional encoder gives a BER value of 0.003125 at the same value of $S / N$ ratio of $10 \mathrm{~dB}$. The BER value in wavelet based OFDM without convolutional encoder can be obtained in wavelet based OFDM with convolutional encoder at $\mathrm{S} / \mathrm{N}$ ratio of $2 \mathrm{~dB}$. The convolutional encoder saves more than $8 \mathrm{~dB}$ which improve the performance than in [9]- [12]. 


\subsection{Proposed Convolutional encoder model}

The last results give an enhancement in the wavelet based OFDM system performance than FFT. The BER value can be reduced when using the Haar function and the convolutional encoder. The BER value in the proposed convolutional encoder model is 0.0005208 at $\mathrm{S} / \mathrm{N}$ ratio of $10 \mathrm{~dB}$ as shown in Fig. 9. The proposed model decreases the value of BER for the same $\mathrm{S} / \mathrm{N}$ ratio used in the above results. When increase the number of stages of the convolutional encoder lead to reducing the BER but increase the complexity and cost. The value of BER with one stage convolutional encoder can be obtained for two stage convolutional encoder at nearly $6.5 \mathrm{~dB}$ to save more than $3.5 \mathrm{~dB}$ which save the system power.

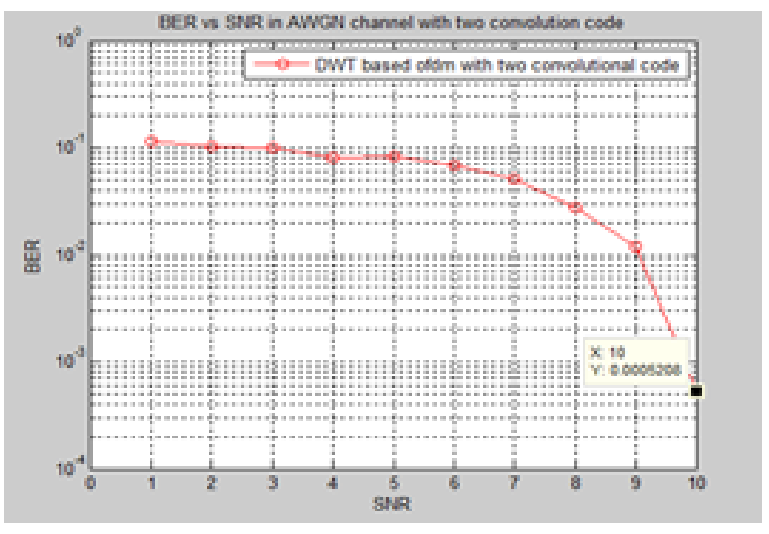

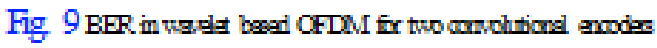

\subsection{Increase the data rates}

The data rates can be increased when using 64-QAM as shown in Fig. 10. The BER has a value of 0.04852 at 10 $\mathrm{dB}$ for 64-QAM [13]. The BER performance is decreased for high data rates. M-QAM modulation gives a better performance and data rates than BPSK and QPSK used in [13].

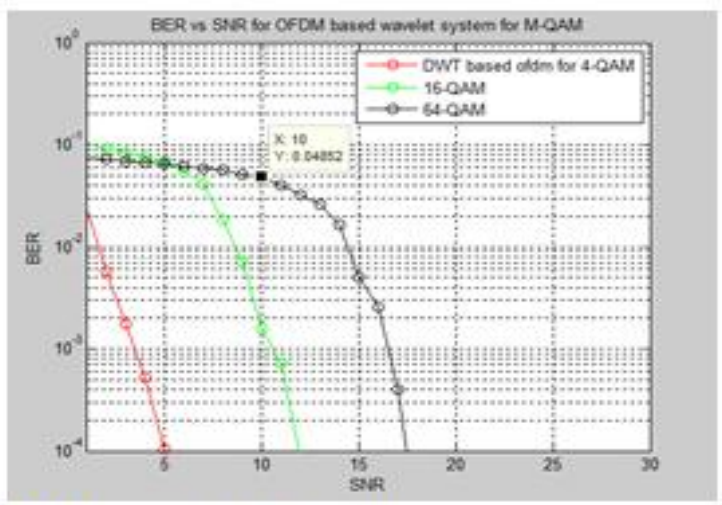

Fig 10 Thergeds bead CFDM firM-QAM

Table 1 will summarize the numerical results with different cases studied
Table 1 Summarized results of the proposed technique

\begin{tabular}{|l|l|l|l|}
\hline The signal to noise ratio & $2 \mathrm{~dB}$ & $5 \mathrm{~dB}$ & $10 \mathrm{~dB}$ \\
\hline $\begin{array}{l}\text { BER in FFT based } \\
\text { OFDM }\end{array}$ & 0.4448 & 0.3516 & 0.1506 \\
\hline $\begin{array}{l}\text { BER at different } \\
\text { wavelet function }\end{array}$ & Haar & DB1 & Rbior \\
\hline $\begin{array}{l}\text { BER Without coding in } \\
\text { wavelet }\end{array}$ & 0.1074 & 0.1049 & 0.08707 \\
\hline $\begin{array}{l}\text { BER with convolution } \\
\text { coding in wavelet 16- } \\
\text { qam }\end{array}$ & 0.0862 & 0.06823 & 0.003125 \\
\hline $\begin{array}{l}\text { BER with two } \\
\text { successive } \\
\text { convolutional code }\end{array}$ & 0.1036 & 0.07995 & 0.0005208 \\
\hline $\begin{array}{l}\text { BER at 64-QAM for } \\
\text { wavelet based OFDM }\end{array}$ & 0.04006 & 0.03998 & 0.02526 \\
\hline $\begin{array}{l}\text { BER at 256-QAM for } \\
\text { wavelet based OFDM }\end{array}$ & 0.06003 & 0.05716 & 0.04635 \\
\hline
\end{tabular}

Comparison between the proposed system and previous works is shown in table 2 [14]-[20]

Table 2 Proposed and previous works

\begin{tabular}{|l|l|l|l|}
\hline $\begin{array}{l}\text { Case } \\
\text { studied }\end{array}$ & $\begin{array}{l}\text { BER in } \\
\text { Proposed } \\
\text { system at } \\
\text { S/N of } \\
\text { 10dB }\end{array}$ & $\begin{array}{l}\text { BER in } \\
\text { previous } \\
\text { works } \\
\text { at 10dB } \\
\text { S/N }\end{array}$ & reference \\
\hline $\begin{array}{l}\text { FFT based } \\
\text { OFDM }\end{array}$ & 0.1506 & 0.636 & {$[14]$} \\
\hline $\begin{array}{l}\text { Wavelet } \\
\text { based } \\
\text { OFDM }\end{array}$ & 0.003125 & 0.1 & {$[15]$} \\
\hline $\begin{array}{l}\text { HAAR } \\
\text { wavelet }\end{array}$ & 0.00151 & 0.0045 & {$[18]$} \\
& & 0.0123 & {$[19]$} \\
\hline $\begin{array}{l}\text { 64-QAM } \\
\text { modulation }\end{array}$ & 0.02526 & 0.1 & {$[20]$} \\
\hline
\end{tabular}

\section{CONCLUSION}

An efficient technique in channel coding is proposed to enhance the performance by decreasing the BER at a certain signal to noise ratio in wavelet based OFDM system. Wavelet based OFDM gives an enhancement in BER performance than FFT based OFDM at the same value of signal to noise ratio 
$\mathrm{S} / \mathrm{N} 10 \mathrm{~dB}$. The proposed channel coding technique gives a BER value of 0.0005208 while a convolutional encoder gives a BER value of 0.0035 for $\mathrm{S} / \mathrm{N}$ ratio of $10 \mathrm{~dB}$. This BER value of the convolutional encoder can be obtained in the proposed technique at $6.5 \mathrm{~dB}$ to save more than $3.5 \mathrm{~dB}$. The proposed channel coding technique operates in 256-QAM with BER nearly equal the BER at 64-QAM which increase the data rates. The proposed technique can reduce the BER value by increasing the convolutional encoder stages which also increase the cost and complexity. The future work for my work will focus on the different encoders than convolutional encoder to give high reduction in BER. The effect of other channel type will also studied in this system as a future work scope.

\section{REFERENCES}

[1] Amit Goyani, Riddhi Shah,"A Review Performance comparison of conventional and wavelet based OFDM system", International Journal of Innovative Science, Engineering \& Technology, Vol. 2 Issue 3, March 2015.

[2] Prof. Siddeeq Y. Ameen and Wa'il A.H. Hadi, "Investigation of Using Turbo Code to Improve the Performance of DWT-OFDM System over Selective Fading Channel", IEEE, 2006.

[3] Karanpreet Kaur , Ankush Kansal," Performance Analysis of Convolutional Interleaved DWT based OFDM system", International Journal of Advanced Research in Computer and Communication Engineering, 2014.

[4] Volkan Kumbasar and Oguz Kucur,“ Alamouti Coded Wavelet based OFDM for Multipath Fading Channels", IEEE,2009.

[5] Juhi Khetrapal, Raj Tiwari,"Improving BER Performance of HAAR and Le-Gall Wavelet Based OFDM", International Journal of Modern Engineering and Research Technology, January 2015.

[6] W. Saad, N. El-Fishawy, S. EL-Rabaie, and M. Shokair,"An Efficient Technique for OFDM System Using Discrete Wavelet Transform”, Springer, 2010.

[7] J. Sudha Rani and K. J. Arvind Chary, "Performance comparison of FFT and DWT based OFDM with Alamouti encoding over Rayleigh fading channel", International Journal of Science and Research (IJSR), 2014.

[8] Ratna Kumari and Prof. P.Subba Rao Garu, “ Haar Wavelet based OFDM system with reduced PAPR for different modulation Techniques Bitra", IJECT and 2014.

[9] Padmaja and Chakri Sreedhar, " BER analysis of conventional and wavelet based OFDM in LTE using different modulation techniques", International hournal of eminent engineering technology, 2015.

[10] Sumi K M, ArunShaji, "Performance comparison of HAAR OFDM and Daubechies OFDM in presence of carrier frequency offset (CFO) and Doppler effect ", International Journal Of Advance Research In Science And Engineering, February 2015.

[11] Santhosh Kumar, B.Vidhya," Bit error rate reduction of DWT based OFDM system", International Journal of Emerging Technology in Computer Science \& "Electronics (IJETCSE) and 2015.

[12] Rakesh, "A Novel approach for efficient bandwidth utilization by employing different multicarrier modulation and MIMO", International Journal of Engineering Research and General Science Volume 3, Issue 3, May-June, 2015.

[13] Gembali Krishna and Adari Satya,"Performance analysis of adaptive MIMO based OFDM using FFT and DWT", international journal of advanced research in science and technology, 2015.

[14] Pratima Manhas, "Performance analysis of DWT based OFDM and FFT based OFDM using various digital modulation techniques and channel coding", International Journal of Computer Applications, 2015.

[15] Rameez Asif, Raed Abd-Alhameed,“A Unique Waveletbased Multicarrier System with and without MIMO over Multipath Channels with AWGN", International Journal of Computer Applications, United Kingdom, 2015.

[16] Sameer A. Dawood and F. Malek, M. S. Anuar, Suha Q. Hadi, "A Proposed Turbo Coded Discrete Multiwavelet Critical-Sampling Transform Based OFDM S" IJET, 2015.

[17] Kavita Trivedi1, Anshu Khare2, Saurabh Dixit3,"BER performance of OFDM with discrete wavelet transform for time dispersive channel", International Journal of Research in Engineering and Technology, 2014.

[18] C. Sreekanth," Performance Evaluation BER of OFDM Decoder Using Different Wavelets", International Journal of Recent Trends in VLSI, Embedded Systems and Signal Processing, 2015.

[19] Ushma.P.pancholi,"a novel technique in channel estimation using wavelet transform in OFDM system", International Journal for Scientific Research \& Development, 2015.

[20] Siddhaling V. Moholkar,"BER Performance for FFT and Wavelet Based OFDM Systems over AWGN Channel" IJRST, 2015. 